EUROTeV-Report-2007-062

Cockcroft-07-15

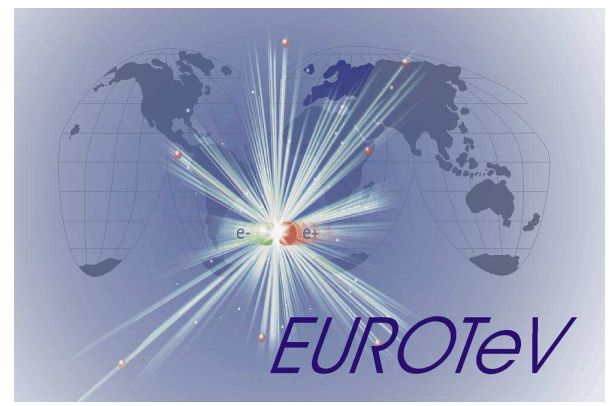

\title{
EMITTANCE GROWTH DUE TO HIGHER ORDER ANGULAR MULTIPOLE MODE WAKEFIELDS IN THE ILC-BDS COLLIMATORS ${ }^{1}$
}

Adriana Bungau and Roger Barlow, The University of Manchester and the Cockcroft Institute, UK

June, 2007

\begin{abstract}
The passage of an off-axis bunch through the collimator gap induces higher order mode wakefields which can lead to emittance growth and consequently can affect the luminosity at the IP - a major concern for the ILC. The emittance growth due to high order angular multipole mode wakefields is calculated and beam profiles at the IP are presented in this paper.
\end{abstract}

\footnotetext{
${ }^{1}$ Work supported by the Commission of the European Communities under the $6^{\text {th }}$ Framework Programme "Structuring the European Research Area", contract number RIDS-011899.
} 


\section{INTRODUCTION}

In a linear collider, the magnets and the cavities are typically misaligned causing the beam trajectory to be offset when it enters the beam delivery system. This can lead to wakefields and dispersive errors which degrade the performance of the collider by causing emittance dilution which in turn causes a reduction of the collider's luminosity. The collimators in the beam delivery system generate short-range wakefields as a result of the interaction between the particle beam and the surrounding environment. Although the wakefields are negligibile for perfectly conducting collimators and ultrarelativistic motion, in the realistic case of finite conductivity and relativistic case they must be taken into account. The longitudinal wakes increase the energy spread while the transverse wakes cause emittance growth [1]. A possible way to relax the wakefields is tapering. But because collimator apertures are tight and particle bunches are close to the collimator edges, the near-wall wakefields play considerable role in single bunch dynamics. For bunches close to the axis, the longitudinal effect is dominated by the monopole mode $(\mathrm{m}=0)$ and the transverse effect is dominated by the dipole mode $(m=1)$. However, when considering near-wall wakefields, higher order modes must be considered and the total wakefield effect is a sum over all multiple contributions [2]. An analytical estimation of the number of modes required for desired accuracy was done by K.Yokoya [3].

The collimator wakefields are separated into two components, a geometric component and a resistive component. The geometric wakefields are due to change in the vacuum chamber section at the collimator, with the walls assumed perfectly conducting. The image charge generated by a charge $q$ displaced by $y$ in a collimator of half gap $a$ is equivalent to a charge $q$ placed at $a^{2} / y$ from the center of the beam axis. When the leading charge reaches the transition, the image charge stops following it and generates an electrostatic field on the particles that are behind the leading particle. The resistive wakes are due to the finite resistivity of the collimator material. For a collimator with tapering sections, both the tapered and the flat sections contribute to the re- sistive wall wakefields because of the finite conductivity of the material.

In this paper, the effects of higher order mode wakefields on beam sizes and luminosity at the IP are presented. Both the geometric and resistive wakefield effects are considered for this study.

\section{WAKEFIELD SIMULATIONS}

Simulations were performed using the MERLIN code [4]. Initially, the standard implementation included only the monopole (longitudinal) and dipole (transverse) wakefields therefore a mathematical formalism for incorporating intrabunch higher order mode wakefields was developed [5]. The current implementation allows one to choose the mode and the appropriate wake function for a specific collimator geometry. For these simulations, the wake function for a steeply tapered collimator with an aperture $a$ placed in a beam pipe with an aperture $b$ was chosen:

$$
W_{m}(z)=2\left(\frac{1}{a^{2 m}}-\frac{1}{b^{2 m}}\right) e^{\frac{-m z}{a}} \theta(z)
$$

where $\theta(z)$ is the unit step function [6]. The ILC-BDS beamline model is based upon the design described in [7]. The current model contains 33 collimators and this comprises spoilers, absorbers, photon masks and protection collimators. The calculations are carried out for a short Gaussian bunch and the beam parameters at the end of the linac are given in Table 1. The beam is injected with an offset at the entrance of the BDS. Although the collimators have rectangular aperture, we use formulae appropriate to apertures with cylindrical symmetry (takind the diameter as the smaller of the vertical and horizontal half gap) as these are avalilable to all orders. The calculations therefore represent estimates rather than exact computations. Nevertheless they should be useful as a guide to what effects are or are not important in maintaining luminosity for the ILC.

\section{GEOMETRIC WAKEFIELDS}

In the absence of wakefields the beam at the IP measures $\sigma_{x}=0.65 \cdot 10^{-6} \mathrm{~m}$ horizontally and $\sigma_{y}=5.69 \cdot 10^{-9} \mathrm{~m}$ vertically. Geometric 
Table 1: The beam parameters at the end of the linac.

\begin{tabular}{|l|c|}
\hline Parameter & Value \\
\hline Bunch charge & $2 \cdot 10^{10}$ \\
Energy & $250 \mathrm{GeV}$ \\
$\beta_{x}, \beta_{y}$ & $45.89 \mathrm{~m}, 10.71 \mathrm{~m}$ \\
$\epsilon_{x}, \epsilon_{y}$ & $2.04 \cdot 10^{-11} \mathrm{~m}, 8.18 \cdot 10^{-14} \mathrm{~m}$ \\
$\sigma_{z}$ & $3.0 \cdot 10^{-4} \mathrm{~m}$ \\
\hline
\end{tabular}

wakefields produce an increase in the beamsize at the interaction region when small offsets are applied to the beam. The offsets are in units of $\sigma_{y}$ where $\sigma_{y}$ represents the vertical beamsize at the begining of the BDS and has the value $\sigma_{y}=0.93 \cdot 10^{-6} \mathrm{~m}$. Figure 1 shows the horizontal beamsize at the IP as a function of beam offset for different wakefield modes. The separation into modes can be noticed from an offset of about $5 \sigma_{y}$ onwards. For just one mode and an offset of $35 \sigma_{y}$ the beam increases to approximately $\sigma_{x}=0.67 \cdot 10^{-6} \mathrm{~m}$ while when four wakefield modes are considered, the beam increases to about $\sigma_{x}=0.7 \cdot 10^{-6} \mathrm{~m}$. The emittance growth in the vertical plane is presented in figure 2 . The split into modes can be seen at offsets of about 10 $\sigma_{y}$. The beamsize increases in the vertical plane to $\sigma_{y}=0.07 \cdot 10^{-6} \mathrm{~m}$ for one mode, while when all four order modes are considered the beamsize increases to $\sigma_{y}=0.19 \cdot 10^{-6} \mathrm{~m}$.

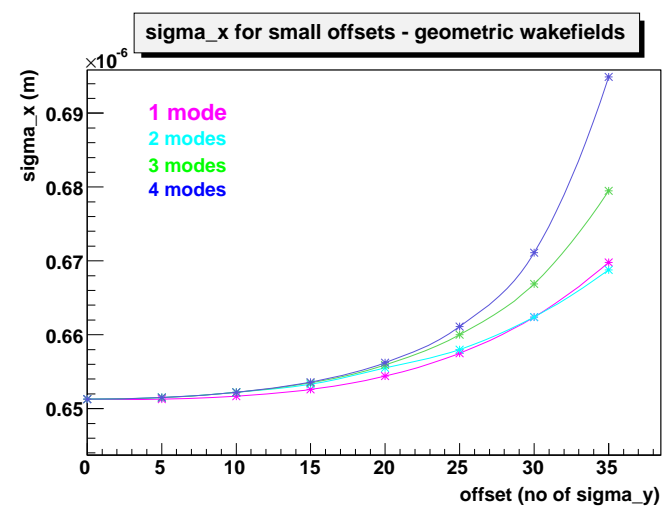

Figure 1: The increase in the horizontal beam size due to higher order mode geometric wakefields.

As a consequence of emittance growth, the luminosity drops with increasing offset and the impact of higher order modes on the luminosity is considerable and has to be taken into account (Figure 3). In absence of wakefields the luminos-

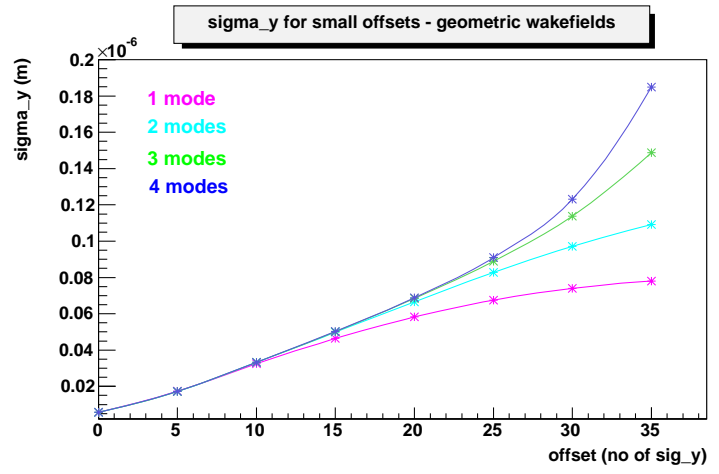

Figure 2: The increase in the vertical beam size due to higher order mode geometric wakefields.

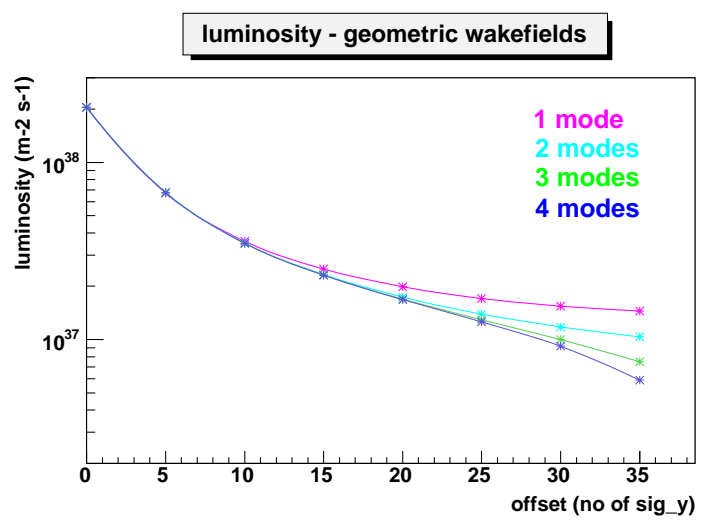

Figure 3: Luminosity loss due to higher order mode geometric wakefields.

ity at the IP is $L=2.03 * 10^{38} \mathrm{~m}^{-2} \mathrm{~s}^{-1}$. Higher order modes produce a decrease in luminosity below $L \sim 10^{37} \mathrm{~m}^{-2} \mathrm{~s}^{-1}$ at $35 \sigma_{y}$ and a drop in luminosity below $L \sim 10^{37} \mathrm{~m}^{-2} \mathrm{~s}^{-1}$ is not acceptable for physics experiments. The luminosity reduction is less than $10 \% \mathrm{n}$ this case (Figure 4).

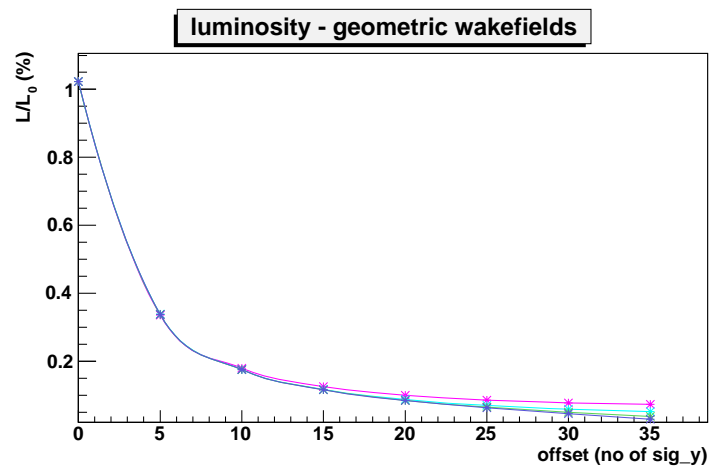

Figure 4: Relative luminosity loss for geometric wakefields. 


\section{RESISTIVE WAKEFIELDS}

The higher order mode resistive wakefields excited in the collimators were studied next. The same beam parameters were used as for the geometric wakefields and the beam was tracked with the same offsets at the begining of the BDS. The collimators were assumed to be made of titanium, with a conductivity $\sigma=2.38 \cdot 10^{6} / \Omega \mathrm{m}$ and the collimator length is the one given in the MAD optics file [7]. The wake function which describes the shock response of the environment to a beam which carries an $m$ moment is [8]:

$$
W_{m}(z)=-\frac{2}{\pi b^{2 m+1}\left(1+\delta_{m 0}\right)} \sqrt{\frac{c}{\sigma}} \frac{L}{\sqrt{z}}
$$

The increase in the horizontal beamsize is presented next (Figure 5). The higher order wakefield modes seem to become significant from offsets greater than $10 \sigma_{y}$. When only one wakefield mode is considered the beamsize is $\sigma_{x}=$ $0.66 \cdot 10^{-6} \mathrm{~m}$ but for a more realistic approach with four wakefield modes, the beamsize is increased to $\sigma_{x}=1.2 \cdot 10^{-6} \mathrm{~m}$ at $35 \sigma_{y}$ beam offset. In the case of vertical beamsize (Figure 6), the split into modes can be seen from offsets of $10 \sigma_{y}$ onwards and at $35 \sigma_{y}$ offset the beamsize increases to $\sigma_{x}=3.4 \cdot 10^{-6} \mathrm{~m}$ for four wakefield modes. This has consequences on luminosity (Figure 7). The luminosity decreases more rapidly than in the geometric case. At $35 \sigma_{y}$ beam offset the luminosity drops to $L \sim 10^{35}$ for four wakefield modes. This corresponds to less than 5\% (Figure 8).

\section{BUNCH DISTORTION}

The transverse wakefields excited in the collimators may damage the beam due to the small aperture of the spoilers and this can also affect the bunch profile at the IP. The bunches may not have a Gaussian shape anymore and they can appear distorted. The distortion is caused by the deflection in angle of the particles that emerge from the collimators which varies with the position along the bunch such that the tail is more affected than the head. Figure 9 shows the bunch shape at the IP due to four wakefield modes after being tracked through the beam delivery system

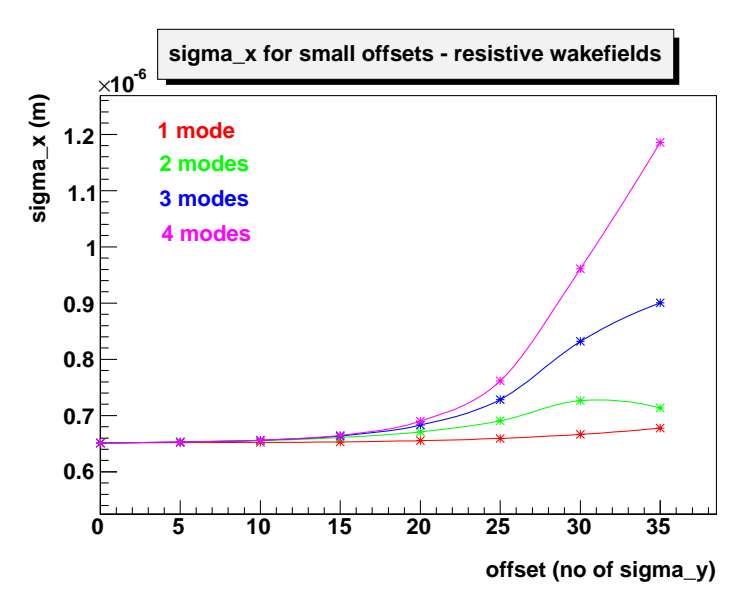

Figure 5: The increase in the horizontal beam size due to resistive wakefields for four modes.

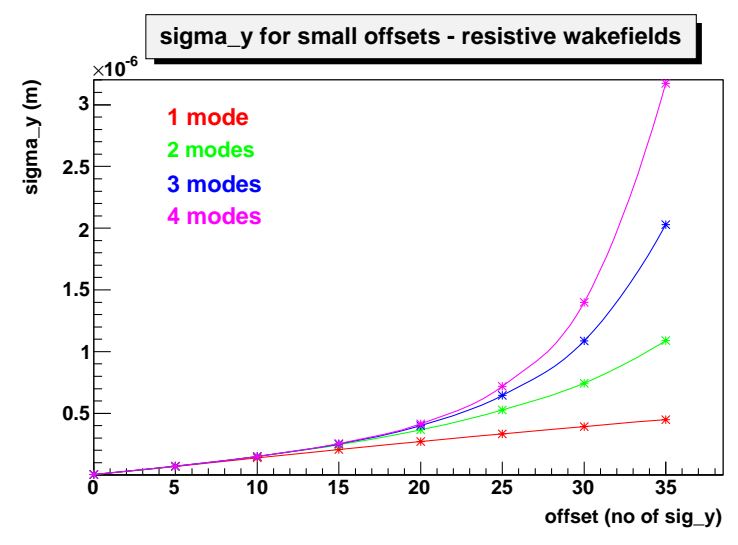

Figure 6: The increase in the vertical beam size due to resistive wakefields for four modes.

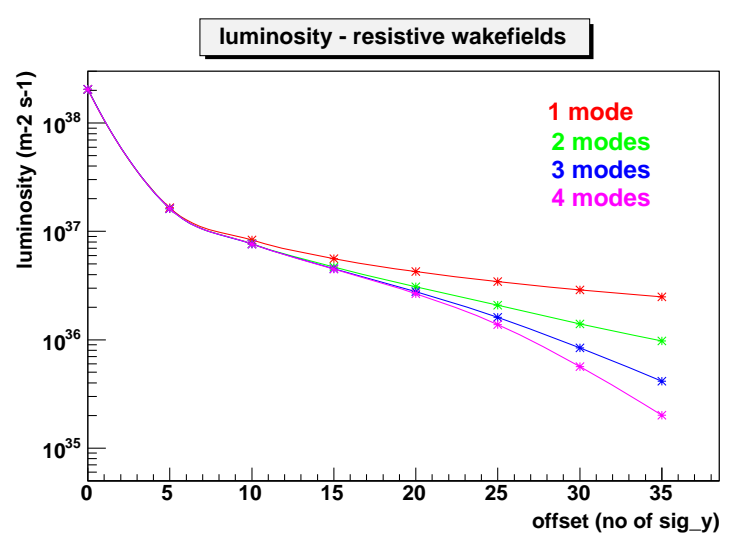

Figure 7: The decrease in luminosity due to resistive wakefields for four modes.

with an offset of $0.45 \mathrm{~mm}$ from the central axis. In this picture one can see that the bunched tail gets a kick while the bunch head is not affected. 


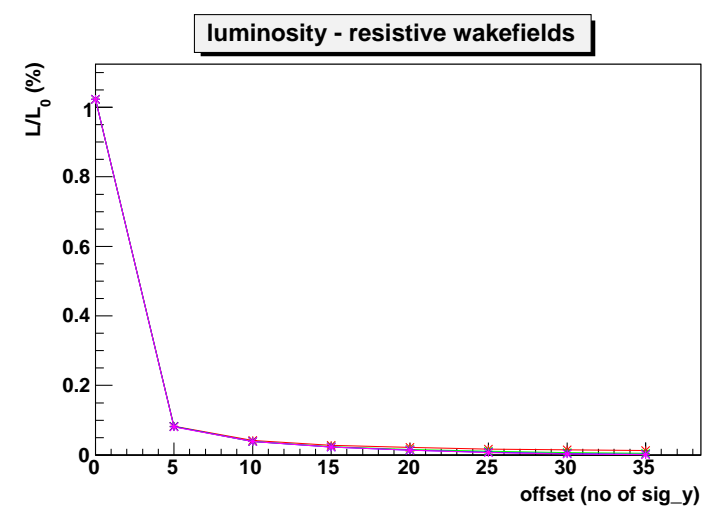

Figure 8: The relative luminosity loss for resistive wakefields.

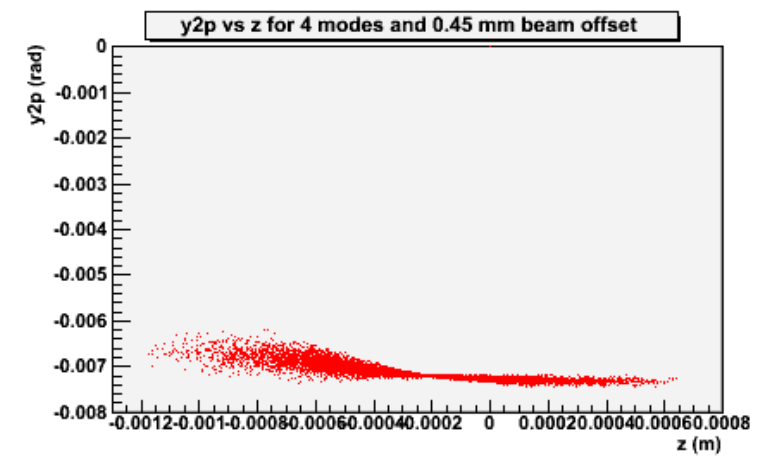

Figure 9: Bunch profile distorted at the IP due to geometric wakefields after tracking through the BDS with an offset of $0.45 \mathrm{~mm}$.

\section{CONCLUSION}

Effects of higher order mode wakefields excited in the ILC-BDS collimators were studied. It was found that for geometric wakefields they become important at larger offsets while at small offsets the effects are dominated by the monopole and dipole mode. At large offsets the luminosity drops to values unacceptable for physics experiments. At small offsets, the decrease in luminosity is not significant.

\section{REFERENCES}

[1] Numerical Calculation of Small-Angle Collimator Wakefields for Short Bunches, I.Zagorodnov, T.Weiland, K.Bane, TESLA Report 2003-18

[2] Near-Wall Wakefields for Optimized Geometry of TTF 2 Collimator, I.Zagorodnov, T. Weiland, M. Dohlus, M.Korfer, TESLA Report 2003-23

[3] Impedance of Slowly Tapered Structures, K.Yokoya, CERN, Geneva, July 1990
[4] MERLIN - A C++ Class Library for performing Charged Particle Accelerator Simulations, http://www.desy.de/ merlin/

[5] Simulation of high order short range wakefields, Adriana Bungau and Roger Barlow, EUROTeV-Report-2006-051

[6] Closed Form Expression for the Geometric Effect of a Beam Scraper on the Transverse Beam Distribution, P.Raimondi et al., SLAC-PUB-8552 (2000)

[7] ILC-BDS optics deck at http://www.slac.stanford.edu/ mdw/ILC/2006b

[8] Physics of Collective Beam Instabilities in High Energy Accelerators, A.Chao, John Wiley and Sons, 1993 